\title{
A Hybrid Genome Assembly Resource for Podosphaera xanthii, the Main Causal Agent of Powdery Mildew Disease in Cucurbits
}

\author{
Álvaro Polonio, ${ }^{1,2}$ Luis Díaz-Martínez,, \\ Diego Romero, ${ }^{1,2}$ Francisco J. López-Ruiz, ${ }^{3}$ and Alejandro Pérez-García ${ }^{1,2, \dagger}$ \\ ${ }^{1}$ Departamento de Microbiología, Facultad de Ciencias, Universidad de Málaga, 29071 Málaga, Spain \\ ${ }^{2}$ Instituto de Hortofruticultura Subtropical y Mediterránea 'La Mayora', Universidad de Málaga, Consejo \\ Superior de Investigaciones Científicas (IHSM-UMA-CSIC), 29071 Málaga, Spain \\ ${ }^{3}$ Centre for Crop and Disease Management, School of Molecular and Life Sciences, Curtin University, \\ Perth, WA 6102, Australia
}

\begin{abstract}
Podosphaera xanthii is the main causal agent of powdery mildew in cucurbits and, arguably, the most important fungal pathogen of cucurbit crops. Here, we present the first reference genome assembly for $P$. xanthii. We performed a hybrid genome assembly, using reads from Illumina NextSeq550 and PacBio Sequel S3. The short and long reads were assembled into 1,727 scaffolds with an $\mathrm{N}_{50}$ size of $163,173 \mathrm{bp}$, resulting in a 142-Mb genome size. The combination of homology-based and ab initio predictions allowed the prediction of 14,911 complete genes. Repetitive sequences comprised $76.2 \%$ of the genome. Our $P$. xanthii genome assembly improves considerably the molecular resources for research on $P$. xanthii-cucurbit interactions and provides new opportunities for further genomics, transcriptomics, and evolutionary studies in powdery mildew fungi.
\end{abstract}

\section{Genome Announcement}

Powdery mildew fungi (Erysiphales, Ascomycota) are pathogens of increasing concern worldwide. Major crops, including cereals, grapevine, and a number of economically important vegetables and ornamentals, are among their hosts (Panstruga and Schulze-Lefert 2002; Seifi et al. 2014; Takamatsu 2013). Powdery mildew fungi are obligate biotrophic parasites that depend on living host cells to survive (Vogel et al. 2004). Obligate biotrophy in fungi is dependent on the development of specialized structures of parasitism-denominated haustoria, whose main functions are the uptake of nutrients from the host and the release of effectors into plant cells (Martínez-Cruz et al. 2014). Owing to their lifestyle as obligate parasites, they cannot be grown on artificial culture media, a fact that has significantly hampered research compared with other filamentous plant pathogens (Fernández-Ortuño et al. 2007). Despite significant progress made in recent decades, powdery mildew diseases continue to be among the most important plant pathological problems worldwide.

Cucurbits are among those economically important vegetables affected by powdery mildews. The disease in cucurbits can be caused by either Podosphaera xanthii or

${ }^{\dagger}$ Corresponding author: A. Pérez-García; aperez@uma.es

A. Polonio and L. Díaz-Martínez contributed equally to this work.

The author(s) declare no conflict of interest.

Accepted for publication 2 November 2020.

\section{Funding}

This study was supported by a grant from the Agencia Estatal de Investigación (AEI) (AGL2016-76216C2-1-R), co-financed by FEDER funds (European Union).

\section{Keywords}

cucurbits, Erysiphales, genomics, metabolomics, Podosphaera xanthii, powdery mildew, proteomics 
Golovinomyces cichoracearum, species that induce identical symptoms but can be easily distinguished by light microscopy (del Pino et al. 2002). Nevertheless, it is widely accepted that $P$. xanthii is the main causal agent of powdery mildew in cucurbits and one of the most important limiting factors for cucurbit production worldwide (Bellón-Gómez et al. 2015; Fernández-Ortuño et al. 2006). In recent years, important efforts have been invested into deciphering the molecular bases of $P$. xanthii pathogenesis. This has produced fundamental resources such as the epiphytic and the haustorial transcriptomes (De Miccolis Angelini et al. 2019; Polonio et al. 2019; Vela-Corcía et al. 2016), and specific tools for the functional analysis of $P$. xanthii genes such as transformation and RNAi silencing protocols (MartínezCruz et al. 2017, 2018a and b). Despite this substantial progress, further research on $P$. xanthii is still needed to uncover novel genes and pathways that will allow us to understand the singularities of this important pathogen of cucurbits. In this study, we present the first draft genome of $P$. xanthii, which was obtained by a hybrid genome assembly approach.

The $P$. xanthii isolate 2086 was cultured onto previously disinfected zucchini cotyledons (Cucurbita pepo L. 'Negro Belleza') (Semillas Fitó, Barcelona, Spain) and maintained in vitro in 8-cm Petri dishes with Bertrand medium under a cycle of $16 \mathrm{~h}$ of light and $8 \mathrm{~h}$ of darkness at $22^{\circ} \mathrm{C}$ for 10 days (Fernández-Ortuño et al. 2006). The isolate was reidentified as $P$. xanthii first by light microscopy (del Pino et al. 2002) before the isolation of genomic DNA and, subsequently, by BLAST analysis of the internal transcribed spacer sequences detected in the genome assembly (100.00\% identity with MT242593.1 and other sequences). Genomic DNA was isolated from $P$. xanthii epiphytic mycelia and conidia collected from powdery mildew-infected zucchini cotyledons and ground with liquid nitrogen in a previously chilled mortar, using the MasterPure Yeast DNA Purification Kit (Lucigen, Middleton, WI, U.S.A.) according to the manufacturer's recommendations. Quality control of DNA samples was carried out using the Nanodrop 2000 spectrophotometer (Thermo Fisher Scientific, Waltham, MA, U.S.A.), 0.6\% agarose gels, and Qubit double-stranded DNA High Sensitivity (HS) Assays Kit in a Qubit fluorometer and expert HS DNA Assays in an Agilent 2100 Bioanalyzer electrophoresis system (Agilent, Santa Clara, CA, U.S.A.).

The extracted DNA was sequenced to obtain both short and long reads using two sequencing platforms, Illumina NextSeq 550 (Illumina, San Diego, CA, U.S.A.) and PacBio Sequel S3 (Pacific Biosciences of California, Inc., Melon Park, CA, U.S.A.). In the case of Illumina, a ready-to-sequence library was constructed using Nextera XT DNA Library Preparation Kit (Illumina) that was sequenced through a midoutput run with 300 cycles $(2 \times$ 150 paired ends) to obtain approximately $300 \times$ coverage, yielding a total of $137,231,749$ paired reads. In the case of PacBio, a 10-kb insert library was constructed using SMRTbell Express Template Prep Kit 2.0 (Pacific Biosciences) that was later sequenced using 1 SMRT cell to obtain a total of $1,787,490$ subreads (mean read length of $8,256 \mathrm{bp}$ ). This strategy led to approximately $15 \times$ coverage of circular consensus sequences (ccs); that is, highly accurate consensus sequences ready for assembly after the process of error correction, corresponding to $185,839 \mathrm{ccs}$ (mean read length of $11,031.56 \mathrm{bp}$ and longest read of $57,662 \mathrm{bp})$.

To estimate the genome size, Illumina paired-end reads were used. Before performing the analysis, the Illumina raw reads were trimmed using SeqtrimNext v2.1.3 (Falgueras et al. 2010) to remove sequences of low quality and low complexity, adapters, and contaminating sequences such as bacterial sequences or host sequences, using, in this case, the zucchini reference genome (Montero-Pau et al. 2018). The k-mer histogram method based on a k-mer distribution with a $k$ value of 21 was performed with KmerGenie v1.7051 (Chikhi and Medvedev 2014), using the preprocessed reads. The main peak obtained corresponded with a $k$ value of 121 , which was used for the estimation of the genome size, obtaining a predicted assembly of $125.58 \mathrm{Mb}$.

The pipeline used to assemble the $P$. xanthii genome and the subsequent gene prediction is shown in Figure 1. The genome assembly was performed using the combination of paired-end preprocessed Illumina reads and PacBio ccs. For this hybrid assembly, several available tools were used. The best results were obtained with MaSuRCA v3.3.4 (Zimin et al. 2013), probably due to the high performance of this software with low-coverage long reads. Illumina paired-end reads were extended into superreads and were mapped to PacBio reads, resulting in megareads. These megareads were converted into contigs and, finally, pairedend reads were reused to do scaffolding and gap repair using the Flye module included in MASuRCA. After that, a new polish step was performed with one round of Pilon v1.23 (Walker 


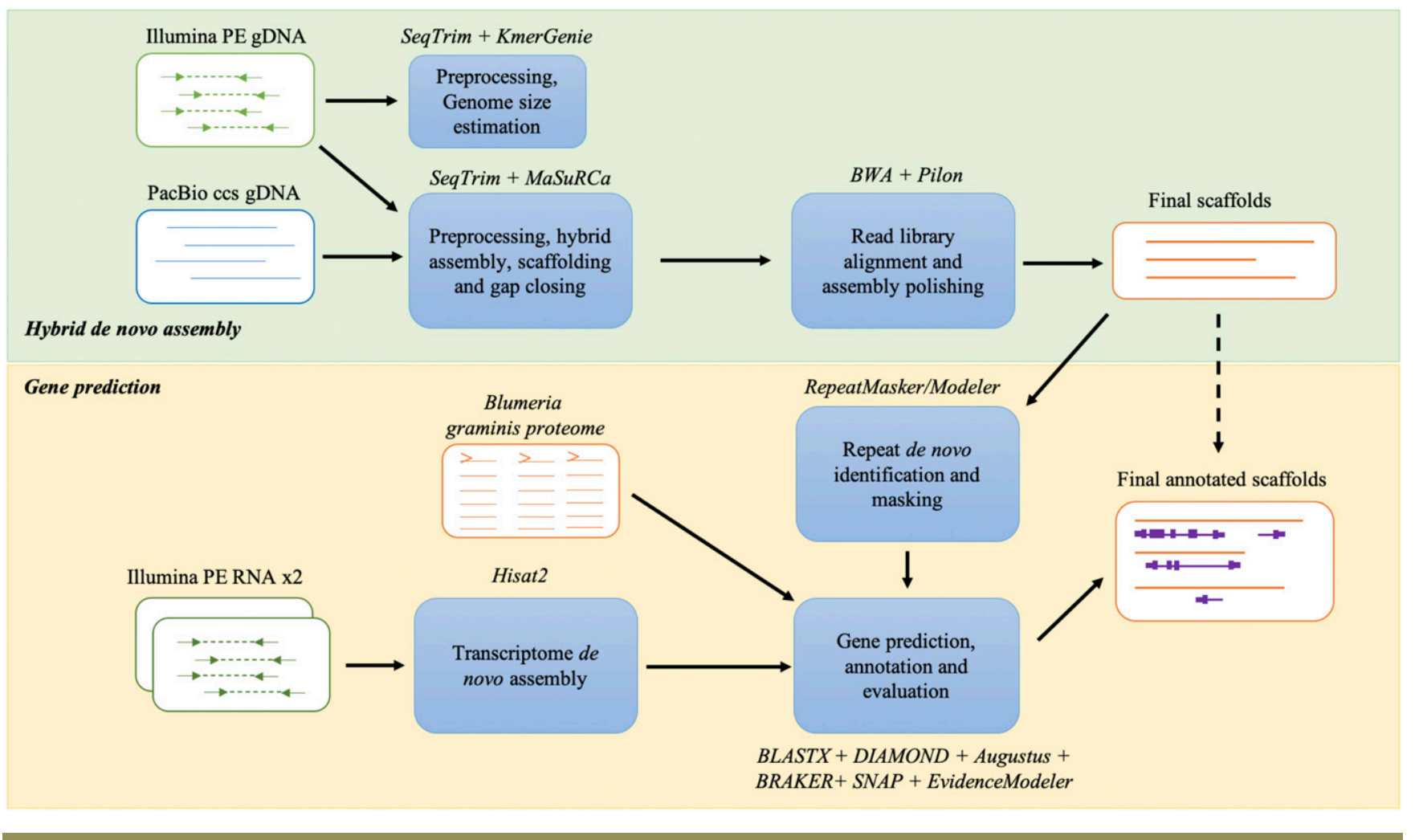

Fig. 1. Pipelines of the Podosphaera xanthii draft genome assembly and gene prediction.

et al. 2014) to fill scaffold gaps using preprocessed reads mapped to the assembly with BWA v0.7.17 (Li and Durbin 2009). The statistics of the genome assembly were carried out using QUAST v.5.0.2 (Gurevich et al. 2013), obtaining 1,727 scaffolds with a final total genome size of $142.11 \mathrm{Mb}$, which was slightly higher than the KmerGenie size estimation (125.58 Mb). The $\mathrm{N}_{50}$ contig length was $163,173 \mathrm{bp}$ and the size of the largest contig obtained was $947,834 \mathrm{bp}$ (Table 1).

The assembled genome of $P$. xanthii was analyzed for repetitive elements prior to gene prediction. To do this, a combination of de novo and homology-based approaches was performed using RepeatModeler v1.0.11 (Flynn et al. 2020) and RepeatMasker v4.0.7 (Tarailo-Graovac and Chen 2009). A large number of repeats were identified that represented up to $76.16 \%$ of the genome (Table 1). Gene prediction from the repeat-masked genome was performed using the GenSAS v6.0 platform (Humann et al. 2019) combining ab initio and homology-based gene predictions. For homology-based prediction, BLAST+ v2.7.1 (Camacho et al. 2009) and DIAMOND v0.9.22 (Buchfink et al. 2015) were used whereas, for ab initio gene prediction, raw reads and cDNA sequences from the epiphytic and haustorial transcriptomes of $P$. xanthii (De Miccolis Angelini et al. 2019; Polonio et al. 2019) and a complete set of protein sequences from Blumeria graminis (Spanu et al. 2010) were used to train Augustus v3.3.1 (Stanke et al. 2008) to predict $P$. xanthii gene models. Augustus identified a total of 22,072 hypothetical genes. Then, SNAP (Korf 2004) and BRAKER v2.1.1 (Hoff et al. 2019) were used to compare and refine the results obtained by Augustus, resulting in a total of 28,014 and 19,590 hypothetical genes, respectively. Finally, EvidenceModeler v1.1.1 (Haas et al. 2008) was used to combine the homology-based and ab initio gene predictions of Augustus, SNAP, and BRAKER into weighted consensus gene structures, yielding 16,030 predicted genes. Among them, 14,911 (93.02\%) were complete genes with standard start and stop codons (Table 1).

The number of predicted genes in the $P$. xanthii genome assembly represents a higher number of genes than previously described in the genomes of other monocot- and dicotinfecting powdery mildews, ranging from 5,854 to 8,470 (Barsoum et al. 2019). However, recently, the genome of the sweet pepper powdery mildew Leveillula taurica presented a predicted gene set of 19,751 (Kusch et al. 2020), which is very similar to $P$. xanthii and other 
Table 1. Core metrics of the draft genome sequence of Podosphaera xanthii

\begin{tabular}{lr} 
Metric & Value \\
Assembly size (bp) & $142,114,041$ \\
Number of scaffolds & 1,727 \\
Number of scaffolds $\geq 1,000 \mathrm{bp}$ & 1,598 \\
Number of scaffolds $\geq 5,000 \mathrm{bp}$ & 1,455 \\
Number of scaffolds $\geq 10,000 \mathrm{bp}$ & 1,382 \\
Number of scaffolds $\geq 25,000 \mathrm{bp}$ & 1,167 \\
Number of scaffolds $\geq 50,000 \mathrm{bp}$ & 873 \\
Largest scaffold (bp) & 947,834 \\
GC content (\%) & 43,23 \\
$\mathrm{~N}_{50}$ (bp) & 163,173 \\
$\mathrm{~N}_{75}$ (bp) & 84,907 \\
$\mathrm{~L}_{50}$ (bp) & 275 \\
L75 (bp) & 574 \\
Number of Ns per 100 kb & 0 \\
Number of predicted genes & 16,030 \\
Number of complete genes (with standard start and & 14,911 \\
stop codons) & \\
Repeat elements (\%) & 76.16 \\
BUSOs (\%) & \\
Complete and single-copy & 94.5 \\
Complete and duplicated & 1 \\
Fragmented & 1.2 \\
Missing & 3.3 \\
\hline
\end{tabular}

obligate biotrophs such as rust fungi, presenting genomes with predicted gene numbers ranging from 13,364 to 28,801 (Barsoum et al. 2019). All of this suggests that, in some dicot powdery mildews, the number of species-specific genes encoded in the genome appears to be higher than in other powdery mildew fungi.

On the other hand, the completeness of the $P$. xanthii genome was studied to carry out a first evaluation of the quality of the genome assembly. For this purpose, BUSCO software v3.0.2 (Simão et al. 2015) was used over the Ascomycota lineage (ascomycota_odb9), which contains a total of 1,315 ortholog groups, using by default Aspergillus nidulans as the model species for Augustus gene predictions. The results showed that a high percentage of Ascomycota core genes were present in the $P$. xanthii genome $(94.5 \%$ as complete and single copy and $1.2 \%$ as fragmented), which was an indicator of the good quality of the genome assembly (Table 1 ).

To conclude, we generated the first-draft genome assembly and ab initio and homologybased gene models for $P$. xanthii. To date, the best-studied powdery mildew genomes are those of the barley and wheat powdery mildew pathogens (Frantzeskakis et al. 2018; Müller et al. 2019; Spanu et al. 2010; Wicker et al. 2013; Wu et al. 2018). To our knowledge, this is the first dicot powdery mildew genome produced using a hybrid assembly approach. This genome considerably improves the resources available for research on $P$. xanthii to date and will likely provide the necessary framework to undertake further investigations on genomics, transcriptomics, and evolutionary biology of powdery mildew fungi.

Data availability. This genome project is indexed at the GenBank database of NCBI under the Bioproject accession number PRJNA646981. All sequencing data were deposited at the Sequence Read Archive database of $\mathrm{NCBI}$ under the accession numbers SRR12260826 (PacBio ccs long reads) and SRR12260825 (Illumina NextSeq550 raw short reads), and the draft genome assembly of $P$. xanthii under the accession number JACSEY000000000.

\section{Acknowledgments}

This study would not have been possible without the computer resources and the technical support provided by the Plataforma Andaluza de Bioinformática of the University of Malaga and, especially, R. Bautista. We thank I. Linares (University of Malaga, Spain) for her 
technical assistance and J. Gómez from Centro de Bioinnovación (University of Malaga) for

the excellent technical support provided in the Illumina sequencing process.

\section{AUTHOR-RECOMMENDED INTERNET RESOURCES}

The Genome Sequence Annotation Server: https://www.gensas.org

SeqtrimNext v2.1.3: http://www.scbi.uma.es/seqtrimnext

\section{Literature Cited}

Barsoum, M., Sabelleck, B., Spanu, P. D., and Panstruga, R. 2019. Rumble in the effector jungle: Candidate effector proteins in interactions of plants with powdery mildew and rust fungi. Crit. Rev. Plant Sci. 38:255-279.

Bellón-Gómez, D., Vela-Corcía, D., Pérez-García, A., and Torés, J. A. 2015. Sensitivity of Podosphaera xanthii populations to anti-powdery-mildew fungicides in Spain. Pest Manage. Sci. 71:1407-1413.

Buchfink, B., Xie, C., and Huson, D. H. 2015. Fast and sensitive protein alignment using DIAMOND. Nat. Methods 12:59-60.

Camacho, C., Coulouris, G., Avagyan, V., Ma, N., Papadopoulos, J., Bealer, K., and Madden, T. L. 2009. BLAST+: Architecture and applications. BMC Bioinf. 10: 421.

Chikhi, R., and Medvedev, P. 2014. Informed and automated k-mer size selection for genome assembly. Bioinformatics 30:31-37.

del Pino, D., Olalla, L., Pérez-García, A., Rivera, M. E., García, S., Moreno, R., de Vicente, A., and Torés, J. A. 2002. Occurrence of races and pathotypes of cucurbit powdery mildew in southeastern Spain. Phytoparasitica 30:459-466.

De Miccolis Angelini, R. M., Pollastro, S., Rotondo, P. R., Laguardia, C., Abate, D., Rotolo, C., and Faretra, F. 2019. Transcriptome sequence resource for the cucurbit powdery mildew pathogen Podosphaera xanthii. Sci. Data 6:95.

Falgueras, J., Lara, A. J., Fernández-Pozo, N., Cantón, F. R., Pérez-Trabado, G., and Claros, M. G. 2010. SeqTrim: A high-throughput pipeline for pre-processing any type of sequence read. BMC Bioinf. 11:38.

Fernández-Ortuño, D., Pérez-García, A., López-Ruiz, F., Romero, D., De Vicente, A., and Torés, J. A. 2006. Occurrence and distribution of resistance to Qol fungicides in populations of Podosphaera fusca in south central Spain. Eur. J. Plant Pathol. 115:215-222.

Fernández-Ortuño, D., Torés, J. A., de Vicente, A., and Pérez-García, A. 2007. Multiple displacement amplification, a powerful tool for molecular genetic analysis of powdery mildew fungi. Curr. Genet. 51:209-219.

Flynn, J. M., Hubley, R., Goubert, C., Rosen, J., Clark, A. G., Feschotte, C., and Smit, A. F. 2020. RepeatModeler2 for automated genomic discovery of transposable element families. Proc. Natl. Acad. Sci. U.S.A. 117:9451-9457.

Frantzeskakis, L., Kracher, B., Kusch, S., Yoshikawa-Maekawa, M., Bauer, S., Pedersen, C., Spanu, P. D., Maekawa, T., Schulze-Lefert, P., and Panstruga, R. 2018. Signatures of host specialization and a recent transposable element burst in the dynamic one-speed genome of the fungal barley powdery mildew pathogen. BMC Genomics 19:381.

Gurevich, A., Saveliev, V., Vyahhi, N., and Tesler, G. 2013. QUAST: Quality assessment tool for genome assemblies. Bioinformatics 29:1072-1075.

Haas, B. J., Salzberg, S. L., Zhu, W., Pertea, M., Allen, J. E., Orvis, J., White, O., Buell, C. R., and Wortman, J. R. 2008. Automated eukaryotic gene structure annotation using EVidenceModeler and the Program to Assemble Spliced Alignments. Genome Biol. 9:R7.

Hoff, K. J., Lomsadze, A., Borodovsky, M., and Stanke, M. 2019. Whole-genome annotation with BRAKER. Pages 65-95 in: Gene Prediction. Methods in Molecular Biology, Vol. 1962. M. Kollmar, ed. Humana, New York, NY, U.S.A.

Humann, J. L., Lee, T., Ficklin, S., and Main, D. 2019. Structural and functional annotation of eukaryotic genomes with GenSAS. Pages 29-51 in: Gene Prediction. Methods in Molecular Biology, Vol. 1962. M. Kollmar, ed. Humana, New York, NY, U.S.A.

Korf, I. 2004. Gene finding in novel genomes. BMC Bioinf. 5:59.

Kusch, S., Németh, M. Z., Vaghefi, N., Ibrahim, H. M. M., Panstruga, R., and Kiss, L. 2020. A short-read genome assembly resource for Leveillula taurica causing powdery mildew disease of sweet pepper (Capsicum annuum). Mol. PlantMicrobe Interact. 33:782-786.

$\mathrm{Li}, \mathrm{H}$., and Durbin, R. 2009. Fast and accurate short read alignment with BurrowsWheeler transform. Bioinformatics 25:1754-1760.
Martínez-Cruz, J., Romero, D., Dávila, J. C., and Pérez-Garcia, A. 2014. The Podosphaera xanthii haustorium, the fungal Trojan horse of cucurbit-powdery mildew interactions. Fungal Genet. Biol. 71:21-31.

Martínez-Cruz, J., Romero, D., de la Torre, F. N., Fernández-Ortuño, D., Torés, J. A., de Vicente, A., and Pérez-Garcia, A. 2018a. The functional characterization of Podosphaera xanthii candidate effector genes reveals novel target functions for fungal pathogenicity. Mol. Plant-Microbe Interact. 31:914-931.

Martínez-Cruz, J., Romero, D., de Vicente, A., and Pérez-García, A. 2017. Transformation of the cucurbit powdery mildew pathogen Podosphaera xanthii by Agrobacterium tumefaciens. New Phytol. 213:1961-1973.

Martínez-Cruz, J., Romero, D., De Vicente, A., and Pérez-García, A. 2018b. Transformation by growth onto agro-infiltrated tissues (TGAT), a simple and efficient alternative for transient transformation of the cucurbit powdery mildew pathogen Podosphaera xanthii. Mol. Plant Pathol. 19:2502-2515.

Montero-Pau, J., Blanca, J., Bombarely, A., Ziarsolo, P., Esteras, C., Martí-Gómez, C., Ferriol, M., Gómez, P., Jamilena, M., Mueller, L., Picó, B., and Cañizares, J. 2018. De novo assembly of the zucchini genome reveals a whole-genome duplication associated with the origin of the Cucurbita genus. Plant Biotechnol. J. 16:1161-1171.

Müller, M. C., Praz, C. R., Sotiropoulos, A. G., Menardo, F., Kunz, L., Schudel, S., Oberhänsli, S., Poretti, M., Wehrli, A., Bourras, S., Keller, B., and Wicker, T. 2019. A chromosome-scale genome assembly reveals a highly dynamic effector repertoire of wheat powdery mildew. New Phytol. 221:2176-2189.

Panstruga, R., and Schulze-Lefert, P. 2002. Live and let live: Insights into powdery mildew disease and resistance. Mol. Plant Pathol. 3:495-502.

Polonio, Á., Seoane, P., Claros, M. G., and Pérez-García, A. 2019. The haustorial transcriptome of the cucurbit pathogen Podosphaera xanthii reveals new insights into the biotrophy and pathogenesis of powdery mildew fungi. BMC Genomics 20:543.

Seifi, A., Gao, D., Zheng, Z., Pavan, S., Faino, L., Visser, R. G. F., Wolters, A. M. A. and Bai, Y. 2014. Genetics and molecular mechanisms of resistance to powdery mildews in tomato (Solanum lycopersicum) and its wild relatives. Eur. J. Plant Pathol. 138:641-665.

Simão, F. A., Waterhouse, R. M., Ioannidis, P., Kriventseva, E. V., and Zdobnov, E. M. 2015. BUSCO: Assessing genome assembly and annotation completeness with single-copy orthologs. Bioinformatics 31:3210-3212.

Spanu, P. D., Abbott, J. C., Amselem, J., Burgis, T. A., Soanes, D. M., Stüber, K., Ver Loren van Themaat, E., Brown, J. K. M., Butcher, S. A., Gurr, S. J., Lebrun, M.-H., Ridout, C. J., Schulze-Lefert, P., Talbot, N. J., Ahmadinejad, N., Ametz, M., Barton, G. R., Benjdia, C., Bidzinski, P., Bindschedler, L. V., Both, M., Brewer, M. T., CadleDavidson, L., Cadle-Davidson, M. M., Collemare, J., Cramer, R., Frenkel, O., Godfrey, D., Harriman, J., Hoede, C., King, B. C., Klages, S., Kleemann, J., Knoll, D., Koti, P. S., Kreplak, J., López-Ruiz, F. J., Lu, X., Maekawa, T., Mahanil, S., Micali, C., Milgroom, M. G., Montana, G., Noir, S., O'Connell, R. J., Oberhaensli, S., Parlange, F., Pedersen, C., Quesneville, H., Reinhardt, R., Rott, M., Sacristán, S., Schmidt, S. M., Schön, M., Skamnioti, P., Sommer, H., Stephens, A., Takahara, H., ThordalChristensen, H., Vigouroux, M., Wessling, R., Wicker, T., and Panstruga, R. 2010. Genome expansion and gene loss in powdery mildew fungi reveal tradeoffs in extreme parasitism. Science 330:1543-1546.

Stanke, M., Diekhans, M., Baertsch, R., and Haussler, D. 2008. Using native and syntenically mapped cDNA alignments to improve de novo gene finding. Bioinformatics 24:637-644.

Takamatsu, S. 2013. Origin and evolution of the powdery mildews (Ascomycota, Erysiphales). Mycoscience 54:75-86.

Tarailo-Graovac, M., and Chen, N. 2009. Using RepeatMasker to identify repetitive elements in genomic sequences. Curr. Protoc. Bioinf. 25:4.10.1-4.10.14.

Vela-Corcía, D., Bautista, R., de Vicente, A., Spanu, P. D., and Pérez-García, A. 2016. De novo analysis of the epiphytic transcriptome of the cucurbit powdery 
mildew fungus Podosphaera xanthii and identification of candidate secreted effector proteins. PLoS One 11:e0163379.

Vogel, J. P., Raab, T. K., Somerville, C. R., and Somerville, S. C. 2004. Mutations in PMR5 result in powdery mildew resistance and altered cell wall composition. Plant J. 40:968-978.

Walker, B. J., Abeel, T., Shea, T., Priest, M., Abouelliel, A., Sakthikumar, S., Cuomo, C. A., Zeng, Q., Wortman, J., Young, S. K., and Earl, A. M. 2014. Pilon: An integrated tool for comprehensive microbial variant detection and genome assembly improvement. PLoS One 9:e112963.

Wicker, T., Oberhäensli, S., Parlange, F., Buchmann, J. P., Shatalina, M., Roffler, S., Ben-David, R., Doležel, J., Šimková, H., Schulze-Lefert, P., Spanu, P. D.,
Bruggmann, R., Amselem, J., Quesneville, H., Ver Loren van Themaat, E., Paape, T., Shimizu, K. K., and Keller, B. 2013. The wheat powdery mildew genome shows the unique evolution of an obligate biotroph. Nat. Genet. 45: 1092-1096.

Wu, Y., Ma, X., Pan, Z., Kale, S. D., Song, Y., King, H., Zhang, Q., Presley, C., Deng, X., Wei, C.-I., and Xiao, S. 2018. Comparative genome analyses reveal sequence features reflecting distinct modes of hostadaptation between dicot and monocot powdery mildew. BMC Genomics 19: 705.

Zimin, A. V., Marçais, G., Puiu, D., Roberts, M., Salzberg, S. L., and Yorke, J. A. 2013. The MaSuRCA genome assembler. Bioinformatics 29:2669-2677. 